\title{
Research on Mobile Cloud Computing Services for Electric Vehicle Charging Facilities ${ }^{1}$
}

\author{
Zhao Yan \\ Beijing Polytechnic,Beijing 100176,China \\ tozhaoyan@sina.com
}

\begin{abstract}
With the development of Internet of things, especially the development of mobile Internet, electric vehicle charging device and location data and power allocation can reach the cloud through mobile Internet, providing reliable basis for public decision-making and public services. However, the provided real-time performance and cost is the key problem of the implementation of the vehicle area network cloud computing. In this paper, aiming at this problem, proposing the mobile Internet scheduling and transmission strategy based on cloud computing, the simulation results show the effectiveness of the proposed method.
\end{abstract}

Keywords: Charging Facilities, Cloud Computing, Data Mining, Public Decision, Mobile Internet

\section{Introduction}

With the rapid development of the world economy, global coal, natural gas, oil and other traditional energy sources become increasingly scarce, the energy environment situation become more and more serious, in order to promote all over the world to accelerate the strategic transformation of transportation energy, setting off a global new round of upsurge development of new energy vehicles. With the development of network technology and vehicle technology, the research and development of electric vehicles in the world are developing continuously, and the new energy vehicles will be the important development trend in the future automobile industry and the whole power technology field. Electric vehicle promotion is a strategic transformation of the traditional fuel automobile industry, electric vehicles broke the traditional car to coal, oil and other fossil fuels, the use of electrical energy as original power of the energy power system, now countries have formed a broad consensus on the development of electric vehicles, the global economy developed areas and famous car manufacturers have to enhance the development of new energy vehicles, governments and major manufacturers are increasing the intensity of investment in human resources, aiming to accelerate the pace of new energy vehicles to market. Early this century, the European Commission announced the new European energy policy, which aimed at reducing the emissions of harmful gases such as automobile exhaust emissions, reducing carbon dioxide and other greenhouse gas emissions, while improving the utilization ratio of solar, wind, hydro and other renewable resources. Electric vehicles in China has also caused a very high value, oil, natural gas and other resource prices have increased the burden on the national economy, the authorities believe that energy has become one of the most important national security issues. The development of non-fossil energy as an important means which to promote the diversification of energy structure, the

\footnotetext{
${ }^{1}$ Foundation project: Research on the Intelligent Navigation System of Mobile Terminal Based the Internet of Things (KM201410858005).
} 
development of electric vehicles as a key to cultivate strategic emerging industries, and promoting the transformation of transport energy ${ }^{[1-3]}$, the reform of the automotive industry power system. The national Ministry of science and Technology launched the "Ten City 1000" large-scale demonstration operations, accelerating the establishment of the electric vehicle industry chain. In the 2008 the Beijing Olympic Games, electric buses as the representative of "Green Olympics", for us to show the new energy vehicles in China's development. From the energy security and environmental protection, China is vigorously developing electric vehicle technology, and strive to shorten the gap with the developed countries in the new opportunities and challenges.

At present, the application of the Internet in electric vehicles has achieved rapid development in China, the Internet of things technology is a typical application, in the operation process of the business model, electric vehicles equipped with GPS locator, in the car driving process to use GPRS wireless network technology to support system to send position status information, so that the background management procedures to monitor the status of electric vehicles in real time, to provide security for the safety of vehicles. Through wireless sensor network, the working status of electric vehicle is acquired by the wireless sensor, and the system is transmitted to the operation support management system through the wireless network. The Internet of things technology is introduced into the electric car industry, which can grasp the operation of electric vehicles in a timely manner. Through the Internet of things technology, in electric vehicles and power stations in various wireless sensor devices, through these sensors and wireless communication technology, in the car and power station to form a small things, to achieve intelligent perception of the power station information, the perceived information through wireless communication technology to the back-end and electric power operation support system, and then to add power station data to shorten the time, thereby reducing the number of infrastructure within the power station. Internet technology development is very fast, it will be the next one the original power to promote the rapid development of the world economy ${ }^{[4-5]}$, the Internet has been applied in all walks of life, it is not only in the smart home, intelligent transportation and industrial detection and other fields to become research and application hotspot in the intelligent transportation industry has become a new research trends. In 2010, LangHejian academician pointed out networking technology is an effective way to solve the traffic problems at China Internet Conference. The development of the Internet of things technology has brought another technological change in the electric vehicle industry, the important direction of the development of electric vehicles in the future will be the Internet of things electric vehicles. Electric motor driven by motor, compared with the traditional fuel consumption of oil and natural gas and other resources, electric vehicle power consumption to obtain power, so greatly reducing the emissions of harmful gases, which is conducive to the improvement of the environment, while easing the country's dependence on oil, electric vehicles will be favored by the technology development in this century. Electric vehicles obtain power by the battery charging, main part of electric vehicles is motor drive control system, which is the basis of the entire electric system, through the electric energy into kinetic energy to get the driving force. However, nowadays there are still many unfavorable factors restricting the expansion of the electric vehicle industry scale, at this stage of the electric car market appeared insurmountable difficulties, electric cars need to charge the battery in the charging station in life, compared with the traditional automobile gas station, electric vehicle charging infrastructure resources are very scarce and the distribution is not uniform, on the other hand, unlike traditional fuel vehicles can drive far mileage, electric car need to rely on electricity to generate power, the electric vehicle charging time is 
long, an electric car need a few hours, the number of battery is much, so there will be trouble charging, battery charging efficiency is low, the battery performance and customer driving safety etc.. At present, in order to solve the problem that the electric vehicle battery charging time is longer, the lease type of the power station mode is rising. Electric vehicles in the power station by the battery quick change device for quick replacement battery saving the time used to charge the battery to improve the efficiency and shorten the service process, but change down battery still need to finish the charging process in power stations, which also can appear the battery scheduling, the concentrated charging of the battery charging equipment requirement is high, when the charging equipment is small, the problem is not obvious, but when the number of vehicles is large, which will appear to meet the demand for a large number of electric vehicles. At the same time, changing electric mode, the model of the electric vehicle battery model need to be unified and standardized. In the process of actual operation, changing model also faces in electric power design input is large, the billing method is not clear, because of the existence of the above problems in electrical operation mode cannot very good, so the two kinds of charging modes have their own problems.

In the mobile Internet era, in addition to mobile phones, laptops, tablet PC can be configured to receive and transmit wireless devices, the future electric vehicles will be configured to receive and transmit equipment, so electric vehicle battery, electric vehicles type and geographic information location can be transmitted to the mobile Internet cloud, while the city ${ }^{[6-8]}$, highway and other locations of electric vehicles, as well as the occupancy of the charge location, the power load of each region can be uploaded to the cloud, Integrated data collected through the mobile Internet cloud, you can determine the charging position of electric vehicles and other functions of the proposed recommendations, and promptly sent to the electric car terminal, or mobile terminal, and can be based on traffic congestion situation, given the most reasonable suggestions. Therefore, based on the mobile Internet cloud computing in the electric vehicle charging application has the economic and practical application, the mobile Internet cloud platform as shown in Figure 1.

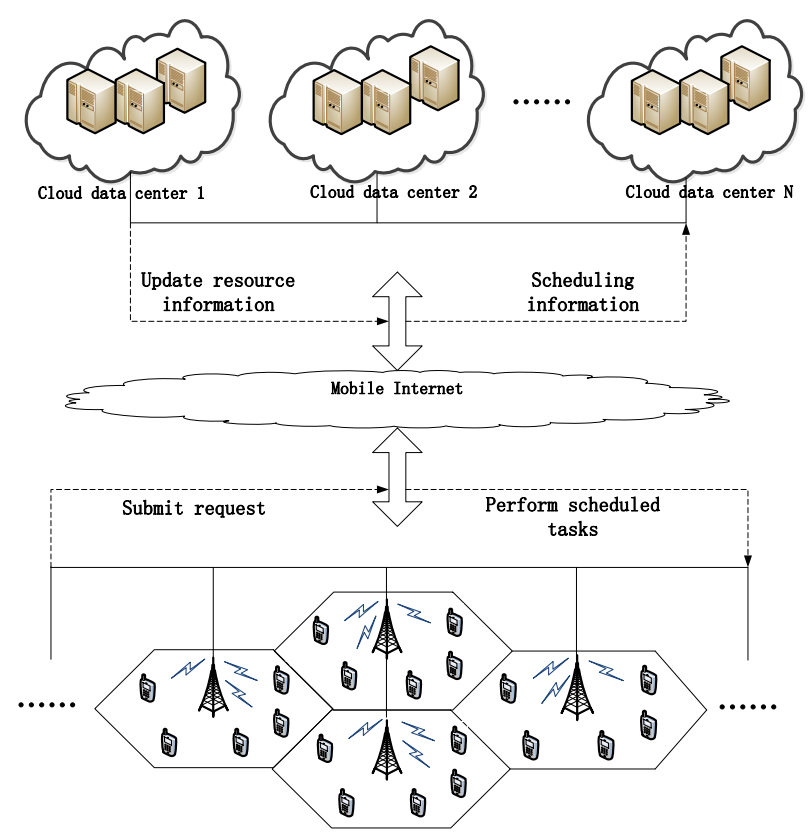

Figure 1. Mobile Internet Topology Map 


\section{Related Works}

\subsection{Electric Vehicle Charging Mode}

From the perspective of the development of electric vehicles, electric vehicles and electric business as a part of the industry, which has a pivotal position, it is necessary for the development of electric vehicles to the market. Electric vehicles mainly depend on the charge and exchange to maintain the operation, and the business will directly affect the development of electric vehicle industry. At present, the main electric vehicle operation mode is divided into the following three types ${ }^{[9]}$.

\section{1) Conventional charging mode}

Conventional charging mode is a typical common mode of electric vehicle charging mode in the energy supply system in the dominant position in the battery power consumption, the use of rechargeable batteries for rechargeable battery charging, the charging mode is not high, full batteries usually need a few hours, and even longer, usually mainly for private cars and work a variety of travel electric car service. The model has little effect on the battery life, the battery life is long, the investment cost of the infrastructure is relatively low, and it is easy to realize.

\section{2) Fast charging mode}

Fast charging mode is relative to the conventional charging mode, the model overcomes the shortcomings that the conventional charging mode charging time. Fast charge using a larger current for electric vehicle charging, compared with conventional mode, charging time is greatly shortened, generally need to wait for tens of minutes. Fast charging mode can meet the needs of some sudden emergency situations, but the fast charging mode adopts high strength current charging, the battery life has a greater impact, and the mode of infrastructure investment requirements will be higher than the conventional charging mode to achieve more conventional formula.

\section{3) Battery faster mode}

Battery faster mode does not charge the battery charging pile, but directly to the electric vehicle on the lack of battery replacement for full battery. In this mode, the electric vehicle operators to achieve the standard of battery leasing and battery type, etc., and the efficiency is very high, the customer is faster down the lack of electric battery unified by the operator to complete the charge. Battery replacement mode service focus is generally public transport, rental, leasing and other groups of vehicles. For electric vehicle service model for the main power of time strictly, such as crude car, bus etc. For electricity mode does not require a charger charging facilities, but it requires a dedicated battery replacement of equipment, battery, battery structure and battery box standardization requirements are higher, another convenient battery quick of changing mode that using leasing operations and need to achieve battery model standardization, at present this hasn't implement very well.

The early electric vehicle power plant, main charging ,charging mode, for a long time, charging efficiency is low, so that customers in the charging station to spend a long time, charging stations will appear the phenomen on of customer waiting in line, which is difficult to meet the needs of emergency, and then put forward a fast charging mode. In the fast charge mode, although the charge time is reduced, but it will bring some new problems, high strength of the current will produce a security risk, and charging technology to put forward higher requirements, but also to improve the way of charging. On the other hand, the current is more than the general assembly of the battery life, and causing the battery mileage is getting shorter, so 
this model is not to promote the application. In view of the various problems existing in the charging mode, charging time is long, the service efficiency is low, and the power station in the middle and later stage of the construction is mainly in the mode of switching mode and charge mode, but there are some defects in this mode. At present, the mode is still in the exploration stage, it requires the customer to buy the bare car, that is, the purchase of electric vehicles do not buy the battery, and the battery used to change the way for the electric operator to change the electric service. In the actual operation process, the model also faces the problem of battery charging scheduling, basic design, billing method is not clear and other issues, at the same time, the battery is bound to be a long time, such as the power station staff to customers to change the model is not consistent with the battery, which is bound to cause a security risk, due to the existence of a variety of problems, for electric operation mode cannot be very good. At present, the electric vehicle industry is still in initial stage, especially in China. It is clear that the electric vehicle charging mode is still in the exploratory stage, although the charging and switching mode has made some progress, but there is still a certain distance from the mass market, and there are some technical difficulties in the actual operation. If you want to be a solid development of electric vehicles and electric business, you need to properly solve these problems.

\subsection{Research Status of Charging Business}

In the intelligent business operation model, the intelligent operation support system includes interface layer, application layer, business layer three stratification. Among them, the interface layer uses the M2M network to realize the interconnection with the station area management gateway, the LAN management gateway, the vehicle area network management gateway. The application layer carry on the comprehensive calculation to the perceived information to the operation support system, and the intelligent service operation assistant data is obtained. Business layer through the intelligent business operations assistant data, through intelligent scheduling based charging, for business, and ultimately to achieve business intelligence. Application layer specific features include: vehicle identification, battery identification, battery detection, vehicle security detection, user identity, account status check, plus power preparation, billing trigger. Vehicle Identification: when the vehicle enters the power station, the vehicle identification RFID receiver automatically and the vehicle area network gateway communications, intelligent identification of the vehicle's identity information, including vehicle VIN number, license plate number, engine number, etc.. Battery identification: when the vehicle enters the power station, the battery inside the station, the RFID receiver automatically and the vehicle domain physical network gateway communication, intelligent identification of the identity of the battery, including battery number, manufacturer, model, etc. Power detection: when the vehicle is in the power station, the power station is detected by the RFID receiver automatically and the vehicle area network gateway communication. Vehicle safety inspection: in the vehicle start state, do the whole of vehicle security detection, including working state of the vehicle, battery status, instrument working state, remaining power, average power consumption, the power consumption and discharge. Safety tips: after the completion of the vehicle safety detection, the detection results are sent to the vehicle terminal, the vehicle driver, to ensure that the driver to understand the safety of the vehicle. User identity: when the vehicle reaches the power station, the RFID receiver of the electric station is automatically associated with the information of the vehicle. Check the status of the account: when the vehicle arrives at the power station and, after the completion of the vehicle identification, identity automatically checks the user's account information, including arrears status, card is frozen and 
other information. Charging position selection: when the vehicle arrives at the power station, after the completion of the vehicle identification, cell recognition, associated with the user identity, the account status check. According to the different types of batteries, power stations charger, charging pile of queuing situation, and then choosing a suitable charging position. Change location choice: when the vehicle reaches the power station, in the completion of vehicle identification, battery identification, user identity, account status check, according to the different types of batteries and power station within the power station waiting for the situation, the choice of the appropriate location for the smart. Charge preparation: when the vehicle reaches the power inside, in the completion of vehicle identification, battery identification, user identity, account status inspection, according to the specific model of the battery, the remaining capacity and other information, select the location and equipment to charge, in advance to do micro grid power transfer related work. Change electric preparation: when the vehicle reaches the power inside, in the completion of vehicle identification, battery identification, user identity, account status inspection, according to the specific model of the battery, remaining capacity and other information, ready to be replaced in a good state of the same type of battery, battery transfer device to transfer to the electric position, so that the battery is ready to change the device. Billing trigger: when the power business is completed, according to the relevant information, including vehicle identity, user level, the power consumption, power and time and other factors, automatically generate billing events, triggering billing collection functions. Business layer specific features include: intelligent security, intelligent charging, intelligent for power. Intelligent Security: security intelligence realized in the running process of the vehicle and do the whole safety of electric vehicle detection, including working state of the vehicle, battery status, instrument working state, remaining power, average power consumption, when electricity consumption, temperature, humidity, law etc., and comprehensive analysis with similar models of historical data security and on vehicle and driver of security early warning, to ensure the safe operation of the electric vehicle. Intelligent charging: intelligent charging from electric vehicles to enter the power station to complete the charging station to complete the entire process of intelligent charging service, including vehicle identification, battery identification, user identity, account information inspection, charging location selection, micro grid power scheduling, charging, billing and triggering, vehicles safety testing process. Smart in changing electric: smart for electric vehicles from entering the power station to complete the power station to complete the entire process of the power station to change the whole process of intelligent service, including vehicle identification, battery identification, user identity, account information inspection, location selection, battery selection, battery delivery, battery quick change, billing trigger, vehicle safety testing process.

Charging in the traditional business mode, when the electric car driving to the power stations, and electric operation staff manual vehicle registration information, battery information and customer information, and then inform with power plant personnel selection and the charging equipment, model matching and selection of artificial evaluate the current position of charging customers usage, selecting line customers less charge position, and then waiting for the customer to add electrical service, noticing to add power plant electric power staff suitable current adjustment for the battery. Charging is completed, cashier personnel by reading the consumption of electricity, which need to deduct the cost of calculation, by placing the customer ID card to the corresponding ID card read-write equipment to complete the billing operations. Adding the whole chariot process, every sub business basically needs staff manual participation, more time consuming, and the charging is 
completed, no interior temperature, voltage, current and working state of equipment safety inspection process, which will inevitably causing the potential safety hazard.

\section{Proposed Scheme}

This paper according to the characteristics of the electric vehicle data, as well as mobile Internet topology characteristics, for the traditional cloud computing data mining is not suitable for mobile Internet cloud platform, according to the characteristics of mobile Internet cloud platform, putting forward a kind of data mining method, and based on it, the configuration method of computational resources and the communication transmission of mobile Internet, cloud computing resource scheduling method, this method can save the communication resources and time; at the same time according to the behavior of users can get the recommended charging scheme.

\subsection{Data Mining based on Electric Charging Data}

For each object of a cluster, $\lambda$ neighborhood contains the number of objects that must be greater than or equal to a given value. If the $\lambda$ neighborhood of an object neighborhood is also satisfied with the above requirements, then the clustering is continued, and the process is repeated until all data objects are processed. Therefore, the density is measured by the number of the $\lambda$ neighborhood of objects, and as long as the object of the area adjacent to the density is more than or equal to a given value to clustering. The clustering process should be divided into several clusters, the process of the completion of the similarity measure, the object in the same cluster have a higher degree of similarity, the similarity between different objects in different clusters, that is, the higher the similarity between the clusters, the better clustering. The similarity between objects is evaluated according to the attribute values of the object, which can be used for the density, distance, connectivity, or concept measurement.

Density is the number of objects in the unit area, and the distance measure is required to be used in the computation, otherwise it is impossible to determine the object in the unit area. The distance similarity measure in clustering is more similar, more nearly.

First, scanning a database and the initial 0-1 matrix is obtained. Then, the "frequent item sets" are generated. The algorithm is described as follows:

Step 1: all processors are scanned in parallel to the database, obtaining the initial " $0-1$ frequent 1 sets" are obtained in this process,

Step 2: removing the unused vector from the initial 0-1 matrix,

Step 3: frequent k-1 sets, and to their own internal connection, generating a set of candidate $\mathrm{k}$,

Step 4: GetLargeltemsets (k) frequent $\mathrm{k}$ itemsets by process,

Step 5: determining whether the $\mathrm{k}$ is empty, if not empty, turning to step 3 , if it is empty, the algorithm ends.

In the first step of the algorithm, all transactions in the database will be evenly distributed to all processors. Each processor scan is assigned to its collection of transactions, and the value of the corresponding position in the initial matrix is obtained. When all the records in the database are scanned, the initial matrix is also obtained. The row of the matrix is represented by a row of data items, the column represents the transaction initialization matrix is generated in accordance with the 
following rules (same as the BOM algorithm first step): if the data item Iteml, Item $2, \ldots$, Itemk exists in the $\mathrm{j}$ transaction, the value of the matrix $A[1, \mathrm{j}], A[2, \mathrm{j}], \ldots$, $\mathrm{A}[\mathrm{k}, \mathrm{j}]$ in corresponding position, which is initialized to 1 , otherwise it is initialized to 0 . Matrix is a $0-1$ matrix.

\subsection{Mobile Cloud Computing Resource Allocation}

The time required for performing the task $t_{i}$ is $T_{t_{i}}$, the amount of computation required for this calculation is $C_{t_{i}}$, the amount of this calculation is calculated as a unit, so it can be obtained from the connection between the two variables according to the formula (1).

$T_{t_{i}}=C_{t_{i}} /\left(P_{s} * \rho_{s}\right)$

Where, $P_{s}$ is computing ability of the sum of the nodes, and $\rho_{s}$ is the proportion which can fulfil the task in the capacity of the node. The time required for the transmission $T T_{t_{i}}$ can be represented by the formula (2).

$$
T T_{t_{i}}=D_{t_{i}} / \beta
$$

So the total processing time can be expressed as type (3)

$P T_{t_{i}}=T_{t_{i}}+\sum_{j=1}^{K} T T_{t_{j}} * s_{j}$

Where represent the nodes that are involved in the allocation and work together to complete the computation tasks have $\mathrm{K}$ nodes, the ratio of the time required by each node $s_{j}$.

Automatic computing resource scheduling and allocation have different optimization objectives, for example, some optimization objectives need to be minimized at the final time of the conditions, the implementation of the cost. This paper defines a scheduling result, $S=(R, \mathrm{M}, \mathrm{TEC}, \mathrm{TET})$ represents a series of distribution free of resources. For a computing task, the mapping of its resources can be divided into the total execution cost, the total execution time. Each resource $r_{i}$ represents the computation resource occupied by the $i$ task.

Each particle is defined by the speed and direction of the search, and each particle is searched according to its optimal solution and local optimal solution. In each search step, the algorithm can be solved according to the speed and direction of the search. For each iteration, the position and speed of its iteration can be determined by the formula (7) and (8).

$$
\begin{aligned}
\mathbf{x}_{i}(n+1)= & \mathbf{x}_{i}(n)+\mathbf{v}_{i}(n) \\
\mathbf{v}_{i}(n+1)= & \lambda \mathbf{v}_{i}(n)+\beta_{1} r_{1}\left(\mathbf{x}_{i}^{*}(n)-\mathbf{x}_{i}(n)\right) \\
& +\beta_{2} r_{2}\left(\mathbf{x}^{*}(n)-\mathbf{x}_{i}(n)\right)
\end{aligned}
$$

Where, $\lambda$ representatives the proportion from the search speed, $r_{1}$ and $r_{2}$ are random number, which used to adjust the direction. $\beta_{1}$ and $\beta_{2}$ represent the acceleration factor, and $\mathbf{x}_{i}^{*}(n)$ represents the optimal solution of the search,, $\mathbf{x}_{i}(n)$ represents the specific position solution of the search.

In the genetic algorithm, the most important is to design the orientation matrix, which is the optimal solution of the optimal solution, and the optimal solution is the 
direction of the orientation matrix, so as to search the optimal solution for the global optimal solution.

In setting orientation matrix, it is important to compare the importance of the two goals, which is to be more close to the global optimum, so as to be able to establish the matrix (9).

$$
C=\left[\begin{array}{ccc}
0 & \cdots & c_{1 n} \\
\vdots & \ddots & \vdots \\
c_{n 1} & \cdots & 0
\end{array}\right]
$$

In order to express the direction, the selection of $-1,0$ and +1 as the matrix element value, if the solution is more close to the optimal solution, then it is +1 , and it is close to the value of 0 , if far from the optimal solution is assigned to -1 . Therefore, only to determine the relationship between them, we can get the orientation matrix, thus ensuring the search direction of the optimal solution is

\subsection{Data Prediction based on User Behavior}

The number of PV (Page View) is the number of Internet users to browse the web is statistics, which is a measure of whether a website is the main indicator of the popular. In the Internet, the user directly through the address bar to enter the URL, or through the search for content, the PV value of the browser to turn to the target link. Because users often access, generally have a high PV value. User's browse interest is divided into the occasional and stable interest. Casual interest is random. Mining stable interest in general is based on Web content and browsing behavior in two ways, the content is realized for the user to browse the web page of repetition and similarity, the behavior of users browse the length of time, pull the number of roll, clicks, etc.. As the browsing behavior involves user privacy, so the following introduction of user interest model based on PV only the main domain name of the site frequency. Improved PV based user interest model analysis of URL's category, the establishment of user interest model. Every day more or less will use the search site, searching the site's PV value is great, and every day with the online office more and more widely, many companies have their own websites and online office platform, employees cannot leave these online office platform, but these sites are not real interest users.

\section{Simulation Results and Analysis}

In this paper, we need to build a cloud simulation platform, which is based on the distributed parallel computing. This platform can be used to store and transmit the data through computer simulation. The experimental results are modified according to the actual situation. This experiment is modified according to the CloudSim. Simulation environment includes computer configuration environment. Its computer simulation environment is shown in Table 1. 
Table 1. VM Configuration

\begin{tabular}{cccc}
\hline & CPU & Memory & Hard Disk \\
\hline$V M_{1}$ & $1 \times 2 \mathrm{GHz}$ & $4 \mathrm{GHz}$ & $500 \mathrm{~GB}$ \\
$V M_{2}$ & $2 \times 2 \mathrm{GHz}$ & $8 \mathrm{GHz}$ & $1 \mathrm{~TB}$ \\
$V M_{3}$ & $4 \times 2 \mathrm{GHz}$ & $16 \mathrm{GHz}$ & $2 \mathrm{~TB}$ \\
$V M_{4}$ & $8 \times 2 \mathrm{GHz}$ & $32 \mathrm{GHz}$ & $4 \mathrm{~TB}$ \\
\hline
\end{tabular}

The simulation of the road is generally shown in the following figure, according to the virtual task and scheduling to achieve the model, the core algorithm for the development of the preparation of its scheduling interval according to the different simulation environment, need to set up a separate.

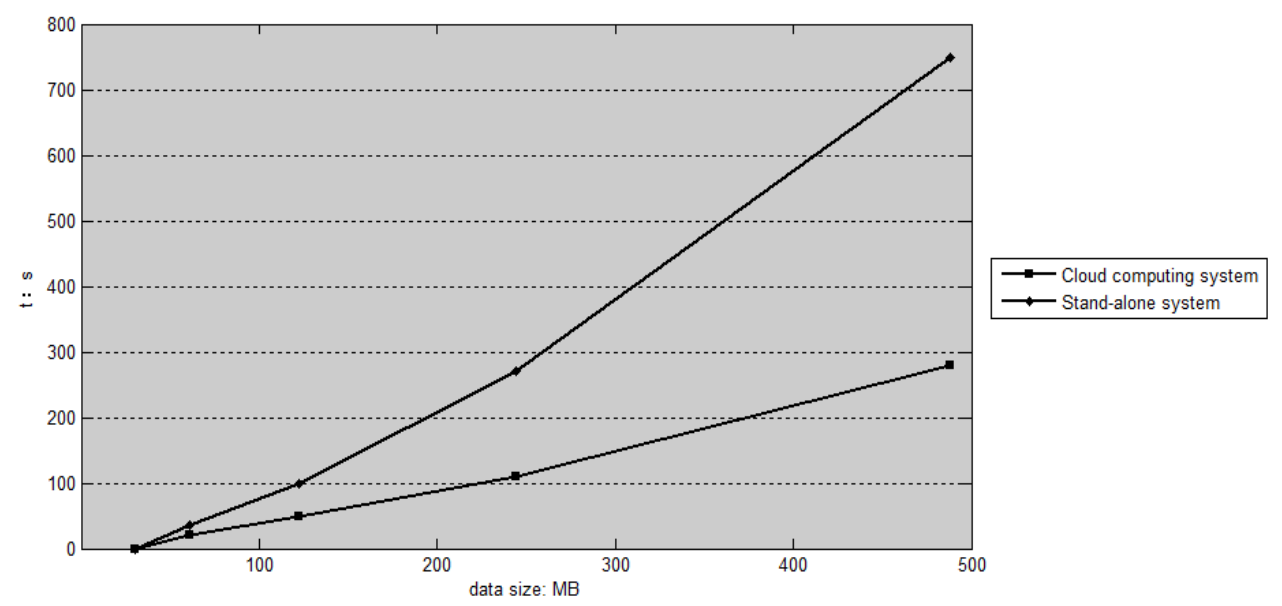

Figure 2. Time Comparison

From Fig.2, it can be seen that the time cost of the algorithm of the single stand mode increases sharply with the increase of sample data, while the time cost of the DBSCAN algorithm increases slowly with the increase of the data. Obviously, the improved algorithm based on cloud computing is more suitable for mass data mining. 


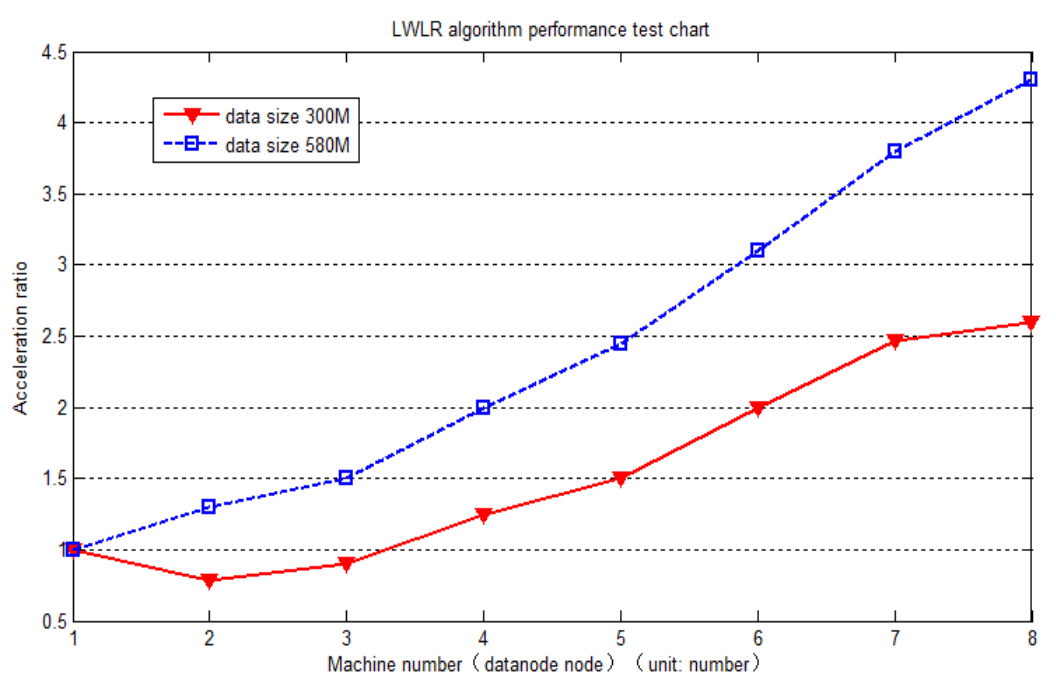

Figure 3. LWLR Algorithm Acceleration Ratio Diagram

Doing the test for different data dimension in stand-alone and cluster, acceleration ratio shows linear correlation basically, just in a single machine and cluster only a datanode cluster as a result of the communication overhead, performance contrast standalone may appear. Through experiment contrast, we can see that with the increase of processing data, the efficiency of parallel computing framework far ahead of single machine processing. We also verified to effectively improve the efficiency of parallel computing system, in fact, in the case of the system hardware to allow, improving the concurrency as far as possibly.

\section{Conclusion}

In this paper, according to the characteristics of the mobile Internet, and the characteristics of the electric vehicle charging, building the data filtering way, in terms of computing resources allocation, this paper adopted cloud computing solutions of the optimal transmission, taking business processing time into account, through the experimental simulation platform, the performance of resource utilization has great improvement, at the same time, the data prediction of user behavior is put forward, and the advantage of the cloud platform is fully played. The experimental results show that the accuracy of the proposed algorithm is higher. Meanwhile, the data mining scheme and the service recommendation scheme based on user's custom are proposed, which provides a basis for the construction of the mobile Internet cloud computing platform.

\section{References}

[1] Rajeev T, Ashok S. Demand management of electric vehicle charging based on a cloud computing framework[C]//Transportation Electrification Asia-Pacific (ITEC Asia-Pacific), 2014 IEEE Conference and Expo. IEEE, 2014: 1-4.

[2] Chaisiri S, Lee B S, Niyato D. Optimization of resource provisioning cost in cloud computing[J]. Services Computing, IEEE Transactions on, 2012, 5(2): 164-177.

[3] Lu Z Y, He Y, Zhao M Y, et al. Design of Electric Vehicle's Operation Management Platform Based on Cloud Computing and Internet of Vehicle[C]//Advanced Materials Research. 2013, 608: 1647-1655.

[4] Whaiduzzaman M, Sookhak M, Gani A, et al. A survey on vehicular cloud computing[J]. Journal of Network and Computer Applications, 2014, 40: 325-344. 
[5] Pawar A, Scholar M T, Kapgate P D. A Review on Virtual Machine Scheduling in Cloud Computing[J]. vol, 2014, 3: 928-933.

[6] Khayyam H, Abawajy J, Javadi B, et al. Intelligent battery energy management and control for vehicle-to-grid via cloud computing network[J]. Applied Energy, 2013, 111: 971-981.

[7] Low C, Chen Y, Wu M. Understanding the determinants of cloud computing adoption[J]. Industrial management \& data systems, 2011, 111(7): 1006-1023.

[8] Mao S W. Virtual Infrastructure Management Framework for Cloud Computing[C]//Proceedings of the 2nd International Conference on Green Communications and Networks 2012 (GCN 2012). Springer Science \& Business Media, 2013, 4: 139.

[9] Di Valerio V, Cardellini V, Lo Presti F. Optimal pricing and service provisioning strategies in cloud systems: a Stackelberg game approach[C]//Cloud Computing (CLOUD), 2013 IEEE Sixth International Conference on. IEEE, 2013: 115-122.

\section{Author}

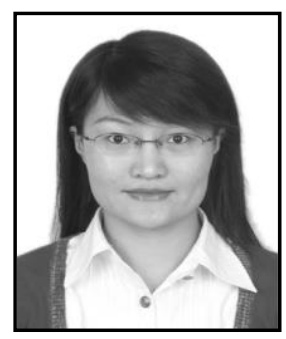

Zhao Yan, She received the Bachelor degree in Computer Science and Technology from Qiqihar University, and the Master degree.in Computer Application Technology from Harbin University of Science and Technology. She is currently researching on the Internet of Things, Mobile Internet and the application and analysis of Big Data. 